[in press, Behavioral and Brain Sciences, September, 2009]

\title{
Are Beliefs the Proper Targets of Adaptationist Analyses?
}

\author{
James R. Liddle and Todd K. Shackelford
}

Florida Atlantic University, Department of Psychology

\begin{abstract}
:
McKay and Dennett's description of beliefs, and misbeliefs in particular, is a commendable contribution to the literature, but we argue that referring to beliefs as adaptive or maladaptive can cause conceptual confusion. "Adaptive" is inconsistently defined in the article, which adds to confusion and renders it difficult to evaluate the claims, particularly the possibility of "adaptive misbelief.”
\end{abstract}

McKay and Dennett (M\&D) open their article by presenting what they consider the "prevailing assumption" (sect. 1, para. 2) of modern evolutionary analyses of belief, namely that true beliefs are adaptive and misbeliefs maladaptive. However, M\&D also present and appear to endorse the content of several quotes (e.g., from Bloom, 2004; Ghiselin, 1974; Haselton \& Nettle, 2006; Stich, 1990) that showcase an alternative evolutionary perspective: beliefs are not relevant to natural selection unless they contributed recurrently to differential reproduction, and there is no reason to assume that only the true beliefs of our ancestors met this criteria. These quotes accurately belie the falsity of what M\&D refer to as the "prevailing assumption" of evolutionary analyses of belief, but this is a relatively minor issue that we do not explore further in this commentary. Instead, we address a more pressing concern: M\&D’s analyses are not based on a coherent definition of "adaptive."

The aim of the target article is to evaluate the assumption that misbeliefs themselves are maladaptive, and to examine candidates for adaptive misbelief. Guided by this aim, it is surprising that M\&D do not provide coherent definitions of the relevant phenomena. Adaptive misbeliefs are loosely defined at various places in the article as beliefs that are "normal" (sect. 5, last para.), "beneficial” (sect. 6, first para.), "aid survival” (sect. 6, first para.), "maximize fitness" (sect. 9, para. 5), "facilitate the negotiation of overwhelming circumstances" (sect. 10, para. 2), "facilitate the successful negotiation of social exchange" (sect. 12, first para.), promote "mental health" (sect. 13, first para.), and "sustain and enhance physical health" (sect. 13, para. 3 , italics in original). M\&D do not explicitly define "adaptive misbelief." Rather, they pose several questions throughout the article in the process of evaluating the plausibility of adaptive misbelief, and these questions imply the sundry definitions we noted. By not providing a 
specific definition of "adaptive," the article lacks a consistent framework for evaluating the candidates of adaptive misbelief.

M\&D acknowledge in a footnote (\# 3) that they conflate conceptually “adaptive” and "adapted” throughout the article, although this acknowledgement does not diminish any confusion, as the reader is left without a specific definition for either term. M\&D also highlight the distinction between psychological adaptation and biological adaptation. These terms are loosely defined with reference to a distinction between "human happiness and genetic fitness" (sect. 10, para. 5), with genetic fitness loosely defined as "having more surviving grandoffspring” (sect. 10, para. 5). The latter definition misses many of the conceptual nuances associated with the concept of fitness from an evolutionary perspective (see Dawkins, 1982). Such an oversimplification is particularly problematic for an article whose arguments hinge on whether beliefs have had an effect on fitness throughout our evolutionary history, which would ultimately determine the status of beliefs as adaptations in and of themselves.

M\&D do obviate several potential confusions throughout their article. For instance, they make a clear distinction between beliefs themselves and the information-processing mechanisms that generate beliefs (sect. 5, last para.). M\&D clearly state that they are interested in the subset of misbeliefs that are generated by properly-functioning cognitive mechanisms, and that these are the candidates for adaptive misbelief. However, M\&D do not justify focusing on beliefs themselves as opposed to the mechanisms that generate beliefs, even though a proper adaptationist perspective (Tooby \& Cosmides, 1992) focuses not on the output of adaptations (e.g., beliefs), but on the design features of the adaptations (e.g., the information-processing mechanisms that generate beliefs). If the information-processing mechanisms of the mind are sensitive to context (Buss et al., 1998), then it is plausible that a belief-generating mechanism can generate true beliefs in one environment and false beliefs in a different environment. Our understanding of why specific beliefs are formed requires an understanding of the mechanisms that generate the beliefs, and referring to beliefs themselves as adaptations obfuscates the importance of the actual adaptations (i.e., the underlying mechanisms).

Despite some conceptual confusion, M\&D present several thought-provoking concepts in the target article. For example, their categorization of misbeliefs in terms of the functioning (or malfunctioning) of the belief formation systems provides an important distinction, although we were surprised to see no reference to Wakefield's (1992) strikingly similar and pioneering evolutionary analyses of dysfunction. We also appreciated the concept of "doxastic shear pins" (sect. 10), which may offer a solid foundation for future empirical and theoretical work on belief formation in extraordinary, psychologically stressful situations. Finally, M\&D's analysis of beliefs suggests an alternative to the proper adaptationist perspective by referring to the output of psychological mechanisms as adaptations. However, the merit of this alternative is difficult to determine, due to the target article's many conceptual confusions.

\section{References:}

Bloom, P. (2004). Descartes' baby: How child development explains what makes us human. Arrow Books. 
Buss, D. M., Haselton, M. G., Shackelford, T. K., Bleske, A. L., \& Wakefield, J. C. (1998). Adaptations, exaptations, and spandrels. American Psychologist, 53, 533-548.

Dawkins, R. (1982). The Extended Phenotype. Oxford, NY: Oxford University Press.

Ghiselin, M. T. (1974). The economy of nature and the evolution of sex. University of California Press.

Haselton, M. G., \& Nettle, D. (2006). The paranoid optimist: An integrative evolutionary model of cognitive biases. Personality and Social Psychology Review, 10, 47-66.

Stich, S. (1990). The fragmentation of reason. MIT Press.

Tooby, J. \& Cosmides, L. (1992). The psychological foundations of culture. In J. Barkow, L. Cosmides, \& J. Tooby (Eds.). The adapted mind: Evolutionary psychology and the generation of culture (pp. 19-136). New York: Oxford University Press.

Wakefield, J. C. (1992). The concept of mental disorder: On the boundary between biological facts and social values. American Psychologist, 47, 373-388. 